\title{
Efficacy, Safety, and Tolerability of an Extended-Release Orally Disintegrating Methylphenidate Tablet in Children 6-12 Years of Age with Attention-Deficit/ Hyperactivity Disorder in the Laboratory Classroom Setting
}

\author{
Ann C. Childress, MD, ${ }^{1}$ Scott H. Kollins, PhD, , Andrew J. Cutler, MD, \\ Andrea Marraffino, $\mathrm{PhD}^{4}$, and Carolyn R. Sikes, $\mathrm{PhD}^{5}$
}

\begin{abstract}
Objective: Methylphenidate extended-release orally disintegrating tablets (MPH XR-ODTs) represent a new technology for MPH delivery. ODTs disintegrate in the mouth without water and provide a pharmacokinetic profile that is consistent with once-daily dosing. This study sought to determine the efficacy, safety, and tolerability of this novel MPH XR-ODT formulation in school-age children with attention-deficit/hyperactivity disorder (ADHD) in a laboratory classroom setting.

Methods: Children aged 6-12 years with ADHD $(n=87)$ were enrolled in this randomized, multicenter, double-blind, placebocontrolled, parallel, laboratory classroom study. The MPH XR-ODT dose was titrated to an optimized dose during a 4-week open-label period and maintained on that dose for 1 week. Participants $(n=85)$ were then randomized to receive their optimized dose of MPH XR-ODT or placebo once daily for 1 week (double blind), culminating in a laboratory classroom testing day. Efficacy was evaluated using the Swanson, Kotkin, Agler, M-Flynn, and Pelham (SKAMP) Attention, Deportment, and Combined scores along with Permanent Product Measure of Performance (PERMP; Attempted and Correct) assessments. Onset and duration of drug action were also evaluated as key secondary endpoints. Safety assessments included adverse events (AEs), physical examinations, electrocardiograms (ECGs), and the Columbia Suicide Severity Rating Scale (C-SSRS).

Results: The average SKAMP-Combined score on the classroom study day was significantly better for the MPH XR-ODT group $(n=43)$ than for the placebo group $(n=39 ; p<0.0001)$. The effect was evident at 1 hour and lasted through 12 hours postdose. The average SKAMP-Attention, SKAMP-Deportment, PERMP-A, and PERMP-C scores were indicative of significantly greater ADHD symptom control for the MPH XR-ODT group. The most common AEs reported were decreased appetite, upper abdominal pain, headache, insomnia, upper respiratory tract infection, affect lability, irritability, cough, and vomiting.

Conclusions: MPH XR-ODT was effective and well tolerated for the treatment of children with ADHD in a laboratory classroom setting.
\end{abstract}

Clinical Trial Registry: NCT01835548 (ClinicalTrials.gov).

Keywords: MPH XR-ODT, ADHD, classroom study, efficacy, safety

\section{Introduction}

A TTENTION-DEFICIT/HYPERACTIVITY DISORDER (ADHD) is the most common neurobehavioral disorder of childhood (Subcommittee on Attention-Deficit/Hyperactivity Disorder et al. 2011) and is characterized by developmentally inappropriate levels of in- attention and/or hyperactivity and impulsivity (Kieling et al. 2010). The prevalence of ADHD in children and adolescents has been reported to be generally similar worldwide-in the range of $5 \%$ to $7 \%$ (Faraone et al. 2003; Polanczyk et al. 2007; Thomas et al. 2015).

The number of children receiving treatment for ADHD has been increasing. There were $27 \%$ more children in the United States

${ }^{1}$ Center for Psychiatry and Behavioral Medicine, Las Vegas, Nevada.

${ }^{2}$ Department of Psychiatry and Behavioral Science, Duke University, Durham, North Carolina.

${ }^{3}$ Florida Clinical Research Center, Bradenton, Florida.

${ }^{4}$ Florida Clinical Research Center, Orlando, Florida.

${ }^{5}$ Neos Therapeutics, Inc., Grand Prairie, Texas.

Funding: This study was supported by funds from Neos Therapeutics, Inc.

(c) Ann C. Childress et al. 2017; Published by Mary Ann Liebert, Inc. This Open Access article is distributed under the terms of the Creative Commons Attribution Noncommercial License (http://creativecommons.org/licenses/by-nc/4.0/) which permits any noncommercial use, distribution, and reproduction in any medium, provided the original author(s) and the source are credited. 
receiving treatment in 2011 than in 2007 (Visser et al. 2014). However, treatment gaps continue to exist; as many as $17.5 \%$ of children with a diagnosis of ADHD were reported by parents as not receiving any treatment in 2011 (Visser et al. 2014). Left untreated, ADHD has an adverse impact on the lives of the affected individuals and their families. ADHD is associated with an increased risk for low self-esteem, social and academic failure, substance abuse, and antisocial and criminal behavior. Parents and siblings may face secondary but significant adverse consequences (Harpin 2005).

Clinical practice guidelines recommend a multimodal approach to treating ADHD, including both stimulant or nonstimulant medications and evidence-based parent- and/or teacher-administered behavior therapy (Pliszka and AACAP Work Group on Quality Issues 2007; Subcommittee on Attention-Deficit/Hyperactivity Disorder et al. 2011; Robb et al. 2014). Either methylphenidate (MPH) or amphetamine, both central nervous system (CNS) stimulants, is recommended as first-line therapy, and they are the most widely prescribed medications for patients with ADHD (Faraone et al. 2006; Pliszka and AACAP Work Group on Quality Issues 2007; Antshel et al. 2011; Robb et al. 2014; Visser et al. 2015). The majority of available dosage forms of stimulants prescribed for ADHD are extended-release (XR) tablets or capsules, which are indicated for once-daily dosing-usually at home during the morning hours (Biederman and Faraone 2005; Faraone et al. 2006; Pliszka and AACAP Work Group on Quality Issues 2007; Robb et al. 2014). XR formulations offer increased convenience and decreased stigma associated with dosing during school hours compared with their immediate-release (IR) counterparts (Feldman and Belanger 2009). Most of the available MPH XR formulations are tablets or capsules (Chavez et al. 2009), which may be difficult for some patients to ingest, especially children (Polaha et al. 2008; Nagaraju et al. 2013). Some MPH XR capsules may be opened and sprinkled on certain foods or dissolved in water or other fluids (Sugrue et al. 2014), although this method requires the entire contents to be ingested immediately after mixing, without being chewed or crushed.

A new orally disintegrating tablet (ODT) formulation of $\mathrm{MPH}$, which provides a pharmacokinetic profile consistent with once-daily dosing, was developed (Childress et al. 2015a). Orally disintegrating tablets, which dissolve in the mouth without water, traditionally have had IR pharmacokinetics (Saharan and Singh 2015); however, this innovative formulation is the first XR-ODT. The XR-ODT formulation is made possible through ion-exchange resin technology. When the MPH salt is dissolved in the presence of the exchange resin, the positively charged mobile ion of the exchange resin $\left(\mathrm{Na}^{+}\right)$ is replaced by the positively charged MPH molecule, resulting in stable MPH microparticles. MPH microparticles are either coated (extended-release) or uncoated (immediate-release) and compressed into ODTs. The coating controls the timing of drug release, enabling a modified release profile as the ODT formulation contains $\sim 30 \%$ IR MPH, and $\sim 70 \%$ XR MPH.

This study was designed to evaluate the efficacy (including onset and duration of effect), safety, and tolerability of MPH XR-ODT compared with placebo in children aged 6-12 years with ADHD in a controlled laboratory classroom setting.

The laboratory classroom paradigm simulates a school classroom and allows for repeated assessments of participants' behavior throughout the day to determine the time course of treatment effects. Classroom studies have been used extensively to evaluate ADHD medication dosing regimens and participants' response as a function of time after drug administration (Wigal and Wigal 2006; Wigal et al. 2013; Robb et al. 2014; Childress et al. 2015b).

\section{Materials and Methods}

\section{Participants}

Male and female children aged 6-12 years meeting the Diagnostic and Statistical Manual of Mental Disorders, 4th edition., Text Revision (DSM-IV-TR) (American Psychiatric Association 2000) criteria for ADHD (any subtype) were enrolled in this trial. The study was approved by a central Institutional Review Board (IRB; Copernicus Group, Research Triangle Park, NC), and each site, if required, submitted the protocol and consent/assent forms to its local IRB. The study was conducted in accordance with Good Clinical Practice and the most recent guidelines of the Declaration of Helsinki (ICH 1996; World Medical Association 2013). Written informed consent and assent were obtained from parents or legal guardians and participants before performing any study-related procedures. Assent was obtained from all participants and documented by the child's signature, or as per local or site requirements.

To be eligible for the study, participants were required to have had a positive response to a stable dose of MPH (20-60 mg of Metadate $\mathrm{CD}^{\circledR}$ [UCB, Inc., Smyrna, GA] or comparable dose of another MPH XR or IR formulation) for at least 1 month before screening, which was confirmed by a positive urine drug test for MPH. The diagnosis of ADHD was confirmed at screening using the Kiddie-Sads-Present and Lifetime version (K-SADS-PL 2009). The participants were required to have a score of $>3$ (mildly ill) on the clinician-administered Clinical Global Impressions-Severity (CGI-S) scale (Busner and Targum 2007), and $>90$ th percentile normative value for gender and age on the ADHD-Rating Scale (ADHD-RSIV) total score, inattentive or hyperactive/impulsive subscales (Childress et al. 2015b) on day 1 after MPH washout (visit 2). The CGI scale is a brief, clinician-rated instrument that measures both the severity of illness and current severity of the patient's overall symptoms (CGI-S) along with the change from the patient's baseline condition (CGI-Improvement [CGI-I]) (Busner and Targum 2007). The ADHD-RS-IV is designed to assess the severity and/or frequency of ADHD symptoms. It is composed of the 18 DSM-IV symptoms of ADHD and is divided into two 9-item subscales; one for hyperactivity/impulsivity and the other for inattention. Each item is scored on a scale of 0 (no or rarely observed symptoms) to 3 (severe or very frequently observed symptoms). The total ADHDRS-IV score ranges from 0 to 54 (Childress et al. 2015b).

Key exclusion criteria included history of poor response, known allergy, serious adverse reactions to any MPH formulation or allergy to any of the components of the MPH XR-ODT, comorbidity that made ADHD diagnosis difficult, need for additional medication to control ADHD symptoms, known medical conditions that would preclude the use of MPH XR-ODT, or history (within the past year) or presence of clinically significant disease or dysfunction that - in the opinion of the investigator-could put the participant at substantial risk or confound study results, current or recent history (within the past year) of drug abuse in the immediate family or by someone living at the participant's home, and positive urine drug screen for other stimulant medications or drugs of abuse at the screening visit.

\section{Study design}

This randomized, multicenter, double-blind, placebo-controlled, parallel group laboratory classroom study was conducted at four sites in the United States. All study sites were experienced with the key assessments used in this study, and rater training was conducted to standardize the rating process across sites. 


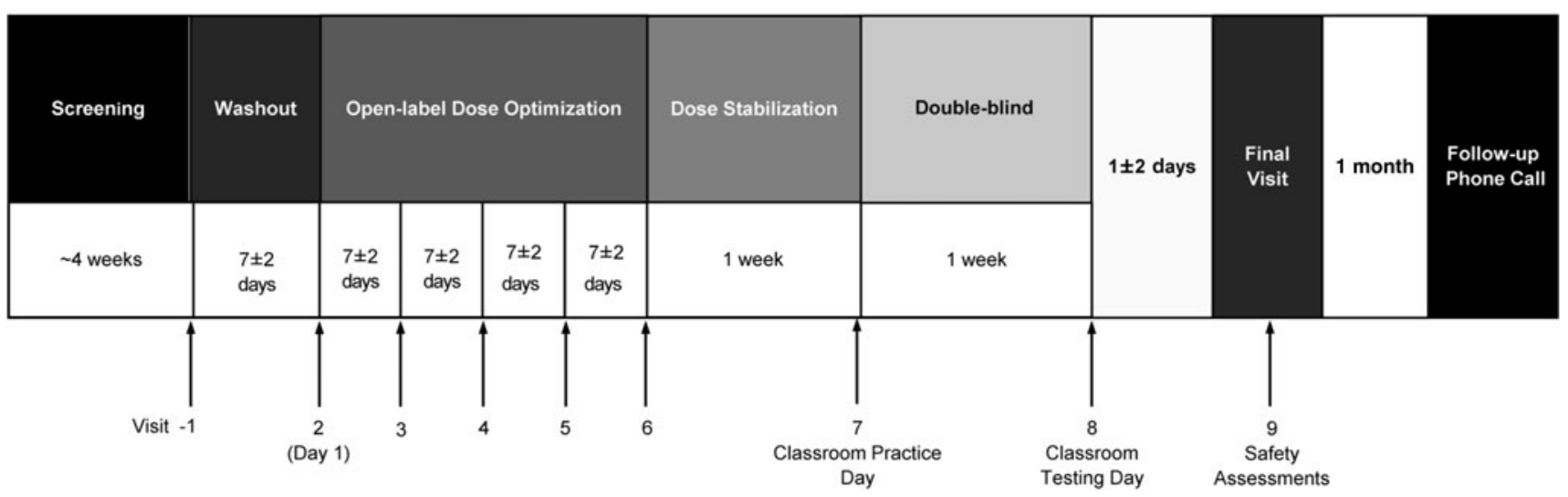

FIG. 1. MPH XR-ODT Laboratory Classroom Study Design. Subjects were screened, underwent drug washout, and then entered an open-label dose-optimization period. Study drug was adjusted during those 4 weeks to the best dose, based on tolerability and efficacy, and then entered a 1-week dose stabilization period. At visit 8, the optimal dose of MPH XR-ODT or placebo was dispensed. MPH XRODT or placebo was administered by study staff at visit 8 . Subjects returned for safety assessments at visit 9 and a follow-up call was completed $\sim 1$ month later. MPH XR-ODT, methylphenidate extended-release orally disintegrating tablet.

The study design is illustrated in Figure 1. The study was divided into five consecutive periods: screening, washout, open-label stepwise dose optimization, dose stabilization, and double-blind, parallel group treatment. The study comprised nine visits and a safety follow-up telephone call.

During the screening visit (study visit 1), participants were assessed for eligibility, inclusion/exclusion criteria, medical/psychiatric history, and prior or current concomitant medications. The KSADS-PL was also used to determine whether any psychiatric comorbidities were present. The Columbia Suicide Severity Rating Scale (C-SSRS; Children's Baseline version) was used to assess the presence or history of suicidal ideation or behavior (Posner et al. 2011). Physical examination, collection of vital signs, 12-lead electrocardiograms (ECGs), clinical laboratory tests (chemistry, hematology, and urinalysis), urine drug screen, and urine pregnancy test (for postmenarcheal female participants) were also performed.

Participants who met screening criteria were instructed to discontinue their current ADHD medication, and, after a washout period of 3-7 days, were reassessed for inclusion/exclusion criteria and a urine drug screen was repeated on day 1 (study visit 2). The C-SSRS (Children's Since-Last-Visit version) assessment was performed to evaluate the presence of suicidal ideation and behavior. The ADHD-RS-IV and CGI-S assessments were completed at this visit to determine ADHD symptomology and establish a baseline for symptom change during the dose optimization phase. In addition, the Permanent Product Measure of Performance (PERMP; Attempted and Correct) placement test was administered to determine the appropriate testing level to be administered during the classroom day. The PERMP placement test consists of eight pages of math problems, ranging from single-digit addition to triple-digit addition and subtraction problems. Subjects have exactly 1 minute to work on each page of 80 problems and are placed at the highest level, at which they are able to complete an average of more than five problems in a minute (Wigal and Wigal 2006).

After the study procedures at visit 2, MPH XR-ODT was initiated at $20 \mathrm{mg}$ once daily the next morning. The parents or caregivers were instructed to place the study drug on the tongue of the child and to allow the drug to disintegrate. The child could move the ODT around in the mouth and swallow the disintegrated drug and saliva when he or she felt the need. Water was allowed ad lib during the study except for 1 hour before through 1 hour postdose, excluding $60 \mathrm{~mL}$ ( 2 fluid ounces) of water for mouth rinsing before drug administration (children rinsed and spit the water into a cup). At each subsequent week of the dose optimization period (visits 3-5), participants were assessed for adverse events (AEs), concomitant medications, vital signs, and ECGs, and the C-SSRS were administered. The dose could be increased in 10-20 mg increments until an optimal dose was achieved or a maximum dose of $60 \mathrm{mg}$ per day was reached based on ADHD-RS-IV and CGI ratings, as well as tolerability.

Individual participants were maintained on their optimized dose for 1 week (dose stabilization period). At the end of this week, subjects participated in a practice laboratory classroom half-day ( $\sim 6$ hours, visit 7 ). This practice half-day familiarizes the participants with the laboratory classroom setting and study procedures that will take place on the double-blind classroom testing day. Participants were then randomized 1:1 to receive either their optimized dose of MPH XR-ODT or placebo once daily during the 1-week, double-blind, parallel-group treatment period. On the last day of the double-blind period (classroom study day; visit 8 ), the participants were confined to the study site for $\sim 14$ hours. At both laboratory classroom sessions, participants were administered the study drug by site staff to ensure precise timing of dosing for predose and postdose assessments completed by trained raters.

Concomitant medications and vital signs were recorded at all visits. AEs were recorded on visit 2 through visit 9. ECGs were obtained at all dose-optimization visits and at the final study visit. Urine drug screens were repeated on the classroom testing day before study drug administration and at the final study visit. Investigators were not provided MPH results until the trial concluded, after the database was locked. Drug adherence was assessed at each visit from visit 3 to visit 8 by recording pill counts of returned medication. The ADHD-RS-IV assessments were performed at each visit during the open-label, dose-optimization, and stabilization periods (visit 2 [baseline] to visit 7 [end of the open-label treatment phase]). The CGI-I assessments were performed at each visit from visit 3 to visit 7 . Efficacy assessments were obtained on the practice and double-blind classroom study days (visits 7 and 8), using the Swanson, Kotkin, Agler, M-Flynn, and Pelham (SKAMP) and the PERMP. 


\section{Efficacy assessments}

The primary endpoint was the SKAMP-Combined postdose score averaged across the seven postdose measurements over the classroom day. The SKAMP ratings were performed on the doubleblind classroom day at baseline (predose), and at 1, 3, 5, 7, 10, 12, and 13 hours postdose. Key secondary endpoints included onset and duration of effect defined as the first time point after dosing, at which MPH XR-ODT separated from placebo on the SKAMP-Combined score, and the last time point at which MPH XR-ODT separated from placebo on the SKAMP-Combined score, respectively.

The SKAMP scale is a validated, 13-item, trained rater assessment of ADHD symptoms in a laboratory classroom setting (Swanson et al. 1978, 1998; Wigal et al. 1998, 2013). The SKAMPCombined rating scale comprises two behavioral subscales: Attention and Deportment. The SKAMP-Combined score is the sum of scores for all 13 items, in which each item is rated from 0 (no impairment) to 6 (maximum impairment); thus, the SKAMPCombined scores range from 0 to 78 -a higher score implies greater impairment (Wigal and Wigal 2006; Wigal et al. 2013). Ratings at each time point were based on the frequency (objective and quantitative counts of specific behaviors) and quality (subjective impressions of overall behavior throughout the rating interval) of participants' behavior by trained raters for 20 minutes of direct observations during each classroom session. Additional secondary endpoints included the SKAMP-Attention, SKAMP-Deportment, PERMP-A, and PERMP-C scores (all averaged over seven postdose measurements). The PERMP is an objective measure of performance that consists of 400 skill-adjusted math problems and is graded as number of problems attempted (PERMP-A) and correct (PERMP-C) in a 10-minute period.

\section{Safety assessments}

Safety assessments included reported and observed AEs, physical examinations, ECGs, vital signs, routine laboratory tests, and the C-SSRS. AEs were elicited at each visit, starting at visit 3. Vital signs and concomitant medications were evaluated at each visit, starting at visit 1. ECGs were completed on visits $1-5$ and visit 9 . Physical examination and laboratory tests were performed at visits 1 and 9. The C-SSRS (Children's Baseline version) assessment was performed at visit 1, and the C-SSRS (Children's Since-Last-Visit version) assessment was performed at each visit, starting at visit 2 .

\section{Statistical analyses}

The primary efficacy analysis was conducted on the full analysis set (FAS, $n=82$ ) using an analysis of covariance (ANCOVA) model with factors for treatment and site, with a covariate for predose SKAMP-Combined score. The FAS consisted of all subjects randomized to treatment who had at least one postdose SKAMPCombined assessment during the classroom testing session on visit 8. All reported primary and secondary efficacy measures in this study refer to the FAS. The sample size was calculated to ensure adequate power for the primary and key secondary endpoints. Based on two previously published studies, the estimated average effect size for the primary efficacy endpoint was 1.1 (Silva et al. 2006; Wigal et al. 2013). A total of 84 participants, or 42 per treatment group, were estimated to provide $>90 \%$ power to detect a treatment difference between MPH XR-ODT and placebo at the significance level of 0.05 (two-tailed, unpaired $t$-test). The $95 \%$ confidence interval (CI) for the difference in least squares (LS) means was estimated using the standard error (SE) of the mean from the ANCOVA.
For key secondary endpoints, a mixed-effects model for repeated measures was used to assess whether the effect of treatment on the SKAMP-Combined score postdose was dependent on the time of assessment postdose. Terms for treatment, site, predose SKAMPCombined score at the classroom testing session, time of assessment postdose, and treatment-by-time interaction were included in this model as fixed effects and subject was included as a random effect. Onset and duration of effect represented ancillary hypotheses within the protocol and were regarded as key secondary endpoints. Specifically, although data from all time points postdosing were compared for descriptive purposes using the said model, formal hypothesis testing commenced at the 5-hour time point. If significance at the $5 \%$ level was achieved at this time point, then the next time point tested was at 3 hours postdose. The testing sequence then proceeded as follows: 7-, 1-, 10-, 12-, and 13-hour time points. If at any time point, significance at the 5\% level was not achieved, formal statistical testing would cease. The onset of effect of MPH XR-ODT (defined as the first time point at which active drug separates from placebo on SKAMP-Combined scores) and duration of effect of MPH XR-ODT (defined as the last time point at which active drug separates from placebo on SKAMP-Combined scores) were estimated using the individual $p$ value for each postdose assessment. A separation was defined as a statistically significant difference at the $5 \%$ level of active drug over placebo.

\section{Results}

\section{Participant disposition and baseline characteristics}

Of the 87 enrolled participants, $65(74.7 \%)$ were diagnosed as combined type using K-SADS-PL, $21(24.1 \%)$ as predominantly inattentive type, and $1(1.1 \%)$ as predominantly hyperactive/impulsive. Eighty-five participants completed the dose optimization period and 83 completed the dose stabilization period. Eighty-three participants were randomized to the double-blind treatment phase and 82 were included in the FAS. The one subject who was excluded from the FAS did not have a baseline SKAMP rating and also had a positive drug screen for amphetamine. Two other subjects were excluded from the per-protocol set because they used prohibited medications. One had been assigned to placebo and the other to MPH XR-ODT.

Demographic and other baseline characteristics were generally similar in the two groups (Table 1). The majority of the enrolled participants were male $(65.9 \%)$, white $(79.3 \%)$, and not Hispanic/ Latino $(65.9 \%)$. The mean (standard deviation [SD]) age of the participants was $9.2(1.75)$ years and body mass index was 18.5 (3.97). No participant was excluded because of nonadherence (defined as taking $<75 \%$ of the study medication between two consecutive visits).

\section{Dose optimization}

During the open-label phase, $12.6 \%$ (11/87), 24.1\% (21/87), $27.6 \%(24 / 87)$, and $33.3 \%$ (29/87) of participants received an optimized dose of $20,30,40$, or $60 \mathrm{mg}$ of the study drug, respectively. The $60 \mathrm{mg}$ dose was the most frequent optimized dose and the distribution was similar in the treatment groups. Subjects included in the FAS had a mean (SD) CGI-S score of $4.5(0.57)$ on day 1 (visit 2$)(n=82 ; 43[52.4 \%]$ had a score of $4 ; 36[43.9 \%]$ had 5 ; and 3 [3.7\%] had 6). At visit 7, the mean CGI-I score was 1.6; 2.4\% (2/ 82 ) of participants were minimally improved; $57.3 \%$ (47/82) were much improved; and $40.2 \%$ (33/82) were very much improved. The mean (SD) ADHD-RS-IV scores for all participants in the safety 
Table 1. Demographic and Baseline Characteristics

\begin{tabular}{|c|c|c|c|}
\hline Parameter & Placebo $(\mathrm{n}=39)$ & $M P H X R-O D T(\mathrm{n}=43)$ & Total $(\mathrm{n}=82)$ \\
\hline \multicolumn{4}{|l|}{ Sex, $n(\%)$} \\
\hline Male & $24(61.5)$ & $30(69.8)$ & $54(65.9)$ \\
\hline Female & $15(38.5)$ & $13(30.2)$ & $28(34.1)$ \\
\hline \multicolumn{4}{|l|}{ Age (years) } \\
\hline Mean \pm SD & $9.3 \pm 1.64$ & $9.1 \pm 1.86$ & $9.2 \pm 1.75$ \\
\hline Median (min, max) & $9.0(6,12)$ & $9.0(6,12)$ & $9.0(6,12)$ \\
\hline \multicolumn{4}{|l|}{ Race, $n(\%)$} \\
\hline White & $31(79.5)$ & $34(79.1)$ & $65(79.3)$ \\
\hline Black or African American & $5(12.8)$ & $5(11.6)$ & $10(12.2)$ \\
\hline Asian & $1(2.6)$ & $1(2.3)$ & $2(2.4)$ \\
\hline Native Hawaiian or Other Pacific Islander & $0(0)$ & $1(2.3)$ & $1(1.2)$ \\
\hline Other & $2(5.1)$ & $2(4.7)$ & $4(4.9)$ \\
\hline \multicolumn{4}{|l|}{ Ethnicity, $n(\%)$} \\
\hline Hispanic/Latino & $13(33.3)$ & $15(34.9)$ & $28(34.1)$ \\
\hline Not Hispanic/Latino & $26(66.7)$ & $28(65.1)$ & $54(65.9)$ \\
\hline \multicolumn{4}{|l|}{ BMI $\left(\mathrm{kg} / \mathrm{m}^{2}\right)$} \\
\hline Mean \pm SD & $18.3 \pm 4.18$ & $18.7 \pm 3.80$ & $18.5 \pm 3.97$ \\
\hline Median (min, max) & $17.0(12.4,30.4)$ & $17.6(13.6,30.5)$ & $17.1(12.4,30.5)$ \\
\hline
\end{tabular}

BMI, body mass index; max, maximum; min, minimum; MPH XR-ODT, methylphenidate extended-release orally disintegrating tablet; $n$, number of participants; SD, standard deviation.

population decreased from visit 2 to visit 7 by 21.4 (8.9). The mean (SD) ADHD-RS-IV scores decreased similarly from visit 2 to visit 7 by 20.4 (8.2) and 22.4 (9.7) for participants who were later either assigned to MPH XR-ODT $(n=44)$ or placebo $(n=41)$ treatment groups, respectively. Improvements in ADHD symptoms during the open-label dose optimization and stabilization periods were not appreciably different for the participants who would later be randomized to either the placebo or the MPH XR-ODT group.

\section{Efficacy assessments}

Primary efficacy measures. The postdose SKAMPCombined scores (LS means [95\% CI]) averaged over the classroom testing day for participants on MPH XR-ODT (14.3 [12.2, 16.4]) were significantly lower (improved) than for participants on placebo $(25.3$ [23.0, 27.6]; $p<0.0001)$ (Table 2). The LS means difference $(95 \% \mathrm{CI})$ was $-11.0(-13.9,-8.2)$.

A sensitivity analysis was performed using a nonparametric model that ranked the average postdose SKAMP-Combined scores. Participants were assigned ranks based on how many SKAMP assessments they completed and reasons for discontinuation along with individual average SKAMP score, if applicable. The following criteria were used to rank participants in ascending worst-to-best order: worst-withdrew during the double-blind phase because of treatment-related reasons; second-worst-withdrew because of nontreatment-related reasons; third-worst-inadequate number of SKAMP scores but completed the classroom visit; fourth-worstaverage SKAMP scores of 60 to 78; fifth-worst-average SKAMP scores of 40 to $<60$; sixth-worst-SKAMP scores of 20 to $<40$; and best-SKAMP scores of $<20$. These rankings were compared between the two treatment groups using ANCOVA for ranked data with factors for treatment group and site, and a covariate for predose SKAMP-Combined score. The model revealed a significant effect $(p<0.0001)$ of MPH XR-ODT on SKAMP-Combined scores, regardless of predose rank main effects or treatment main effects.

Secondary efficacy measures. The onset and duration of efficacy were assessed by comparing the SKAMP-Combined scores for participants on MPH XR-ODT versus placebo at 1, 3, 5, $7,10,12$, and 13 hours postdose on the classroom study day. The MPH XR-ODT-treated group demonstrated significantly lower scores than placebo at 1 hour postdose (LS means difference [95\% CI]); -10.7 [-13.6, -7.9]; $p<0.0001$; Fig. 2). The difference between the two groups continued to be statistically significant at each assessment through 12 hours postdose $(p<0.0001$ at 3,5 , and

Table 2. Average Postdose SKamP Scores Indicate MPH XR-ODT Improved ADHD Symptoms

\begin{tabular}{|c|c|c|c|c|}
\hline & SKAMP-Combined score in $M P H X R-O D T$ & SKAMP-Combined score in placebo & Difference & $\mathrm{p}$ \\
\hline LS mean $(95 \% \mathrm{CI})$ & $14.3(12.2,16.4)$ & $25.3(23.0,27.6)$ & $-11.0(-13.9,-8.2)$ & $<0.0001$ \\
\hline & SKAMP-Attention score in $M P H X R-O D T$ & SKAMP-Attention score in placebo & & \\
\hline LS mean $(95 \% \mathrm{CI})$ & $7.7(6.7,8.7)$ & $12.2(11.1,13.4)$ & $-4.5(-5.9,-3.1)$ & $<0.0001$ \\
\hline \multicolumn{5}{|c|}{ SKAMP-Deportment score in MPH XR-ODT SKAMP-Deportment score in placebo } \\
\hline LS mean $(95 \% \mathrm{CI})$ & $6.7(5.2,8.1)$ & $12.8(11.3,14.3)$ & $-6.1(-8.0,-4.3)$ & $<0.0001$ \\
\hline
\end{tabular}

SKAMP-Attention, Deportment, and Combined scores were measured on the classroom study day, predose, and at several postdose time points (1,3,5,7,10, 12 , and 13 hours) for participants in the full analysis set $(n=82)$ on MPH XR-ODT or placebo. A lower SKAMP score indicates less symptomatology. The SKAMP scores, averaged over all postdose assessments, were significantly lower for MPH XR-ODT than for placebo, implying improved ADHD symptoms.

ADHD, attention-deficit/hyperactivity disorder; CI, confidence interval; LS, least squares; MPH XR-ODT, methylphenidate extended-release orally disintegrating tablet; SKAMP, Swanson, Kotkin, Agler, M-Flynn, and Pelham rating scale. 


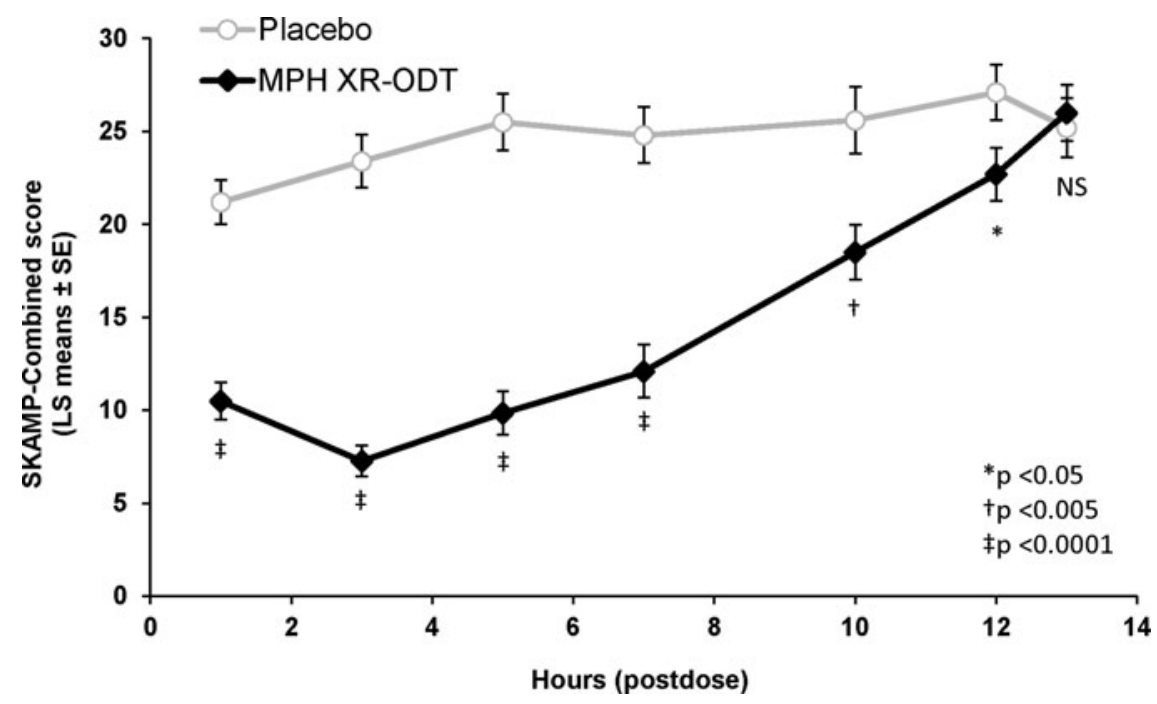

FIG. 2. The Separation of Between-Group SKAMP-Combined Scores over the Classroom Day. SKAMP-Combined scores were measured predose and at seven postdose time points for participants in the full analysis set $(n=82)$. Data from all time points postdosing were compared for descriptive purposes using repeated measures ANCOVA on the postdose scores. ANCOVA, analysis of covariance; LS, least squares; MPH XR-ODT methylphenidate extended-release orally disintegrating tablet; NS, non-signficant; SE, standard error of the mean; SKAMP, Swanson, Kotkin, Agler, M-Flynn, and Pelham rating scale.

7 hours; $p=0.0024$ at 10 hours; and $p=0.0262$ at 12 hours; Fig. 2). The SKAMP-Combined predose score (mean [SD]) for the MPH XR-ODT group (26.8 [11.5]) was significantly higher than that for the placebo group (19.1 [11.0]; $p<0.0001)$, a phenomenon that has been observed in multiple previous classroom studies (Wigal et al. 2013). There was no significant site main effect $(p=0.12)$. The difference in the predose SKAMP scores was included in the model evaluating the efficacy of MPH XR-ODT compared with placebo.

The SKAMP-Attention and SKAMP-Deportment scores on the classroom study day were secondary endpoints. The average LS means postdose SKAMP-Attention score for the MPH XR-ODT group (7.7 [95\% CI: 6.7, 8.7]) was significantly lower than that for the placebo group $(12.2[11.1,13.4] ; p<0.0001)$, and the LS means difference was $-4.5(-5.9,-3.1)$ (Table 2). Similarly, the average LS means postdose SKAMP-Deportment score for the MPH XRODT group $(6.7$ [5.2, 8.1]) was significantly lower than that for the placebo group $(12.8[11.3,14.3] ; p<0.0001)$, and the LS means difference was $-6.1(-8.0,-4.3)$.

Similar to SKAMP-Combined score, the SKAMP-Attention and SKAMP-Deportment scores showed efficacy of MPH XR-ODT as early as 1 hour postdose (LS means difference [95\% CI]: SKAMPAttention; -4.0 [-5.6, -2.3] and SKAMP-Deportment; -7.2 [-9.3,$5.2]$; both $p<0.0001)$. Efficacy continued through 12 hours postdose for SKAMP-Attention $(-3.4[-5.2,-1.5]$ at 12 hours, $p=0.0005)$ and through 10 hours for SKAMP-Deportment $(-4.0[-6.9,-1.1]$ at 10 hours, $p=0.0075)$. The mean (SD) SKAMP-Attention and SKAMP-Deportment scores predose were significantly higher for the MPH XR-ODT group than for the placebo group (SKAMPAttention: 12.6 [4.5] and 8.4 [4.3] for MPH XR-ODT and placebo groups, respectively; SKAMP-Deportment: 14.2 [8.9] and 10.7 [8.0], respectively; $p<0.0001$ for both). There was no significant site main effect for SKAMP-Deportment score $(p=0.63)$, but this effect was significant for SKAMP-Attention score $(p=0.0098)$.

Both PERMP-A and PERMP-C average postdose scores on the classroom study day showed significant treatment effects (LS means [95\% CI]; PERMP-A: 111 [102, 119] and 79.3 [70.3, 88.2] for MPH XR-ODT and placebo, respectively; PERMP-C: 107
$[98.9,116]$ and 75.7 [67.0, 84.4], respectively; $p<0.0001$ for both). Similar to SKAMP scores, the onset of effect was evident in PERMP-A and PERMP-C scores at 1 hour postdose $(p<0.0001$ for both), which continued for up to 12 hours ( $p<0.01$ at 12 hours for both scores, Fig. 3). The mean (SD) PERMP-A and PERMP-C scores predose were significantly different in the MPH XR-ODT and placebo groups (PERMP-A: 87.0 [53.7] and 93.5 [42.5] for MPH XR-ODT and placebo, respectively; PERMP-C: 83.8 [53.6] and 90.5 [43.5], respectively, $p<0.0001$ for both), but there was no significant site main effect $(p>0.85)$.

\section{Safety results}

Of the 87 enrolled participants, 85 were randomized to receive the study medication during the double-blind period. All 87 participants were exposed to MPH XR-ODT for a mean (SD) of 38.7 (5.23) days (MPH XR-ODT group: 42.6 [1.35] days, $n=44$; placebo group: 35.5 [1.29] days, $n=41)$. All 87 participants were exposed for an average of 12.6 (10.90) days to MPH XR-ODT $20 \mathrm{mg} /$ day; 75 participants were exposed for 13.1 (9.93) days to MPH XRODT $30 \mathrm{mg} /$ day; 55 participants were exposed for 14.7 (8.93) days to MPH XR-ODT $40 \mathrm{mg} / \mathrm{day}$; and 30 participants were exposed for 17.9 (4.27) days to MPH XR-ODT $60 \mathrm{mg} /$ day. The mean (SD) daily dose of MPH XR-ODT that all participants received during the dose optimization/stabilization periods was 34.3 (9.06) $\mathrm{mg}$. In addition, the participants in the MPH XR-ODT group received their optimized daily dose for 1 week during the double-blind period.

During the dose optimization/stabilization periods, $80.5 \%$ of the participants (70/87) experienced a total of $170 \mathrm{AEs}$, whereas during the double-blind period, $24.4 \%$ of the participants (10/41) on placebo and $25 \%$ of the participants (11/44) on MPH XR-ODT experienced 13 and 11 AEs, respectively. Of the 170 AEs experienced during the dose optimization/stabilization phase, 126 were considered by the investigators to be related to the study drug. Of the 13 AEs experienced by subjects who received placebo during the double-blind phase, 2 were considered by the investigators to be related to the study drug. Of the 11 AEs experienced by subjects 


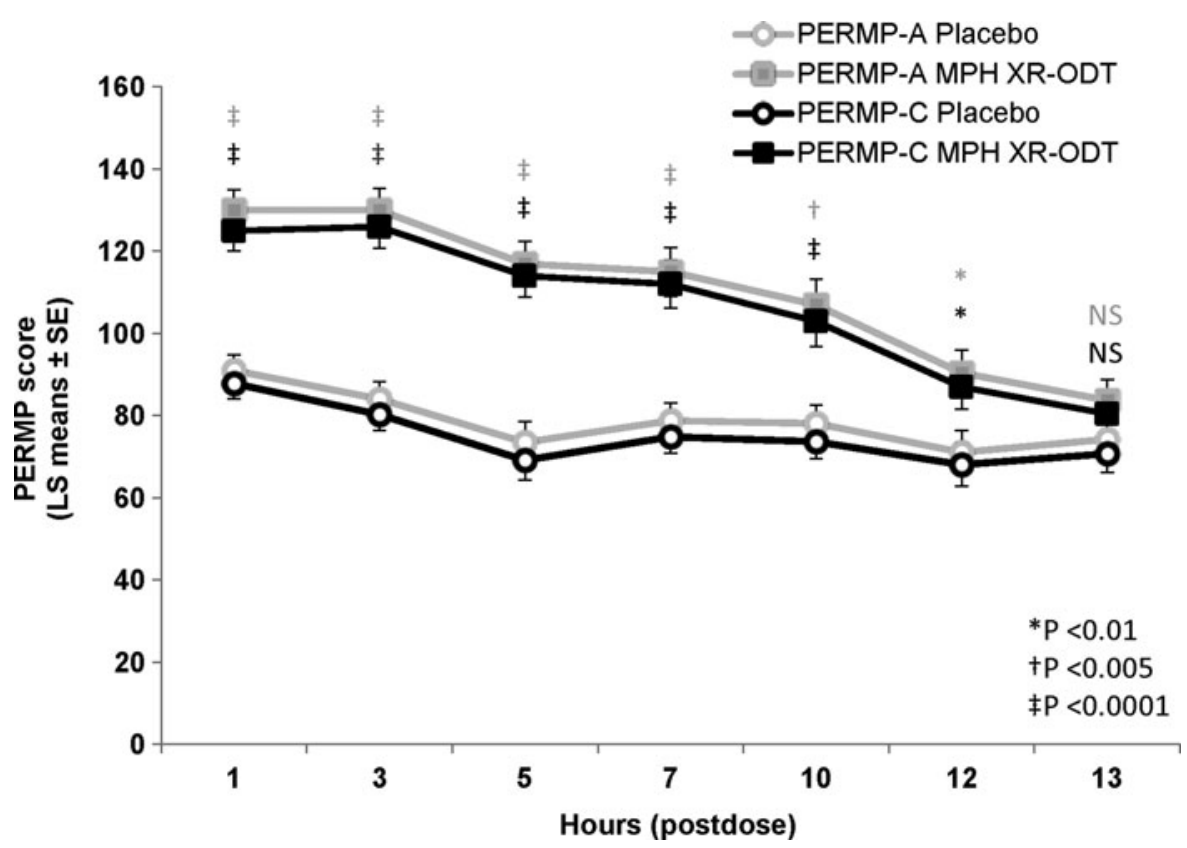

FIG. 3. MPH XR-ODT Improved PERMP-A and PERMP-C Scores. PERMP-A and PERMP-C scores were measured predose and at seven postdose time points for participants in the full analysis set $(n=82)$ on MPH XR-ODT or placebo $(p$ values: gray=PERMP-A, black=PERMP-C). LS, least squares; MPH XR-ODT, methylphenidate extended-release orally disintegrating tablet; NS, non-significant; PERMP-A, permanent product measure of performance-attempted; PERMP-C, permanent product measure of performance-correct; SE, standard error of the mean.

who received MPH XR-ODT during the double-blind phase, 2 were considered by the investigators to be related to the study drug.

The most common (occurred in $>5 \%$ of the participants) AEs during the open-label dose optimization/stabilization periods were decreased appetite, upper abdominal pain, headache, insomnia, upper respiratory tract infection, affect lability, irritability, cough, and vomiting (Table 3 ). The only AE that occurred in $>5 \%$ of participants during the double-blind period was upper respiratory tract infection (Table 3). Of the AEs occurring during the doubleblind phase in all participants, only one instance of mild tricho-

Table 3. Most Common ( $>5 \%$ ) Treatment-Emergent AdVERSE EVEnTs

\begin{tabular}{|c|c|c|}
\hline Adverse event, $\mathrm{n}(\%)$ & \multicolumn{2}{|c|}{$\begin{array}{c}\text { Optimization/ } \\
\text { stabilization periods }(\mathrm{n}=87)\end{array}$} \\
\hline Decreased appetite & \multicolumn{2}{|c|}{$23(26.4)$} \\
\hline Upper abdominal pain & \multicolumn{2}{|c|}{$19(21.8)$} \\
\hline Headache & \multicolumn{2}{|c|}{$17(19.5)$} \\
\hline Insomnia & \multicolumn{2}{|c|}{$11(12.6)$} \\
\hline Upper respiratory tract infection & \multicolumn{2}{|c|}{$10(11.5)$} \\
\hline Affect lability & \multicolumn{2}{|c|}{$8(9.2)$} \\
\hline Irritability & \multicolumn{2}{|c|}{$6(6.9)$} \\
\hline Cough & \multicolumn{2}{|c|}{$5(5.7)$} \\
\hline \multirow[t]{3}{*}{ Vomiting } & $5(5$ & \\
\hline & \multicolumn{2}{|c|}{ Double-blind period } \\
\hline & $\begin{array}{c}M P H X R-O D T \\
(\mathrm{n}=44)\end{array}$ & $\begin{array}{l}\text { Placebo } \\
(\mathrm{n}=41)\end{array}$ \\
\hline Upper respiratory tract infection & $4(9.1)$ & $3(7.3)$ \\
\hline
\end{tabular}

MPH XR-ODT, methylphenidate extended-release orally disintegrating tablet, $n$, number of participants. tillomania and one instance of headache were assessed by the investigator as possibly related to MPH XR-ODT.

The majority of AEs during the double-blind phase were considered mild. There were one severe AE (influenza in one participant on placebo) and two moderate AEs (upper respiratory tract infection in one participant on MPH XR-ODT; abnormal liver function test in one participant on placebo). None of the moderate or severe AEs were assessed by the investigator to be related to the study drug. There were two discontinuations because of AEs: one participant discontinued during the optimization/stabilization period (upper abdominal pain) and one participant discontinued during the double-blind period (severe influenza, placebo group). There were no clinically significant abnormal laboratory values, vital signs, end-of-study physical examinations, or ECG findings. None of the participants exhibited suicidal ideation or behavior, as assessed by C-SSRS ratings, at any study visit. There were no serious AEs (SAEs) or deaths during the study.

\section{Discussion}

The results of this study demonstrate the efficacy, safety, and tolerability of MPH XR-ODT in the laboratory classroom study. As assessed by the SKAMP-Combined score measured on the classroom study day, MPH XR-ODT significantly reduced ADHD symptom severity compared with placebo. The onset of response was evident as early as 1 hour after administration, and efficacy was maintained through 12 hours postdose. In addition, MPH XRODT showed efficacy across multiple secondary endpoints, including SKAMP-Attention, SKAMP-Deportment, PERMP-A, and PERMP-C scores on the classroom study day. MPH XR-ODT also showed efficacy on the ADHD-RS-IV and the CGI scales during the dose optimization and stabilization periods, although these were not specifically identified $a$ priori as efficacy endpoints. 
The baseline (predose) SKAMP scores for the participants receiving MPH XR-ODT were higher than in those receiving placebo on the classroom testing day as has been reported previously in similar studies assessing CNS stimulants, including another MPH XR formulation (Wigal et al. 2009, 2013). This is an interesting and reproducible finding, and it is interesting to speculate that this might be explained by a rebound effect from the previous day's MPH wearing off in the participants treated with MPH XR-ODT.

The most common AEs during the entire study period were generally mild and consistent with the mechanism of action for MPH (Punja et al. 2013; Ramtvedt et al. 2014). No deaths or SAEs were reported during the study.

To our knowledge, this is the first study describing an MPH XRODT formulation in any therapeutic area. Well-validated tools were used to measure ADHD symptoms in a parallel design laboratory classroom setting to measure the efficacy and safety of MPH XRODT as compared with placebo. Since participants with any of the three clinical subtypes of ADHD were eligible for enrollment, these results may be applicable to children with all types of ADHD seen in clinical practice. The MPH XR-ODT technology presents a novel alternative treatment option for adults and children with ADHD.

There are limitations to this study. The duration was relatively short and the study enrolled only children aged 6-12 years without comorbidities that could potentially confound study results, consistent with other phase 3 trial designs. Therefore, results may not be relevant for some patients who have ADHD. In addition, only participants with a previous history of response to MPH were included after being washed out of their current treatment before receiving MPH XR-ODT. Further trials should be conducted to determine the long-term efficacy and safety of MPH XR-ODT in patients who have ADHD and to establish its efficacy and safety in other populations of patients with ADHD, including preschool children (ages 4-5 years) and patients with a history of positive response to either class of stimulant. The American Academy of Pediatrics guidelines recommend evidence-based parent- and/or teacher-administered behavior therapy as first-line treatment in preschool children. However, MPH is recommended if the behavior interventions do not result in significant symptom improvement (Subcommittee on Attention-Deficit/Hyperactivity et al. 2011).

\section{Conclusions}

This was the first clinical efficacy and safety study of an XRODT formulation for the treatment of ADHD. Using multiple validated instruments (SKAMP and PERMP assessments), MPH XR-ODT was shown to improve ADHD symptoms in children aged 6-12 years. The onset of the effect of MPH XR-ODT was at 1 hour after administration, and the effect lasted through 12 hours. Although it is difficult to draw conclusions between formulations without head-to-head comparisons, this MPH XR-ODT appears to have a duration of efficacy similar to that of other MPH XR products compared with placebo in laboratory classroom studies. Both osmotic release oral system MPH (Concerta ${ }^{\circledR}$ Janssen Pharmaceuticals, Inc., Titusville, NJ) and MPH XR oral suspension (Quillivant $\mathrm{XR}^{\circledR}$ NextWave Pharmaceuticals, Inc., Cupertino, CA) had a duration of effect of 12 hours in laboratory classroom trials (Swanson et al. 2003; Wigal et al. 2013).

As expected, the drug was well tolerated; children who had tolerability issues with their previous MPH medication were excluded from the study. In addition, participants were titrated to an optimized dose based on both efficacy and tolerability. The majority of AEs reported or observed during the study were mild and consistent with the mechanism of action for MPH. Only one subject discontinued during the dose optimization phase because of an AE thought to be related to $\mathrm{MPH}$. No AEs were related to changes in vital signs.

\section{Clinical Significance}

MPH is a frequently prescribed, well-established treatment option that is effective in controlling ADHD symptoms. Therefore, ADHD patients may benefit from an alternative MPH formulation that does not require swallowing an intact capsule or tablet. MPH XR-ODT offers an efficacy profile consistent with once-daily dosing and disintegrates in the mouth without water.

\section{Acknowledgments}

Editorial assistance was provided by Narender Dhingra, MBBS, $\mathrm{PhD}$, and Jennifer Tyson, $\mathrm{PhD}$, of AlphaBioCom, King of Prussia, PA, and supported by Neos Therapeutics, Inc., Grand Prairie, TX.

\section{Disclosures}

Dr. A.C.C. has received research support from, consulted with, acted as an invited speaker for, and/or served on advisory boards for Alcobra Pharma, Arbor Pharmaceuticals, Forest Research Institute, Ironshore Pharmaceuticals, Lilly USA, Lundbeck, Neos Therapeutics, Neurovance, NextWave Pharmaceuticals, Noven Pharmaceuticals, Otsuka Pharmaceutical, Pfizer, Purdue Pharma, Rhodes Pharmaceuticals, Shire Pharmaceuticals, Sunovion Pharmaceuticals, Theravance Biopharma, and Tris Pharma.

Dr. S.H.K. has received research support from and/or consulted with Akili Interactive; Alcobra Pharma; Arbor Pharmaceuticals; Atentiv; National Institutes of Health (NIDA, NIEHS, NICHD); Neos Therapeutics, Neurovance; Purdue Pharma; Rhodes Pharmaceuticals; Shire Pharmaceuticals; Sunovion Pharmaceuticals; Tris Pharma; and US Environmental Protection Agency.

Dr. A.J.C. has received research support from, consulted with, and/or acted as an invited speaker for Akili Interactive; Arbor Pharmaceuticals; AstraZeneca; Janssen Pharmaceuticals; Lilly; Lundbeck; Neos Therapeutics; Neurovance; Novartis Pharmaceuticals; Noven Pharmaceuticals; Otsuka Pharmaceutical; Pfizer; Purdue Pharma; Rhodes Pharmaceuticals; Shire Pharmaceuticals; Sunovion Pharmaceuticals; Supernus Pharmaceuticals; Takeda Pharmaceuticals; and Teva Pharmaceutical.

Dr. C.R.S. is an employee of Neos Therapeutics and has stock options. Dr. Sikes has stock in Pfizer.

Dr. A.M. has no financial relationships to disclose.

\section{References}

American Psychiatric Association: Diagnostic and Statistical Manual of Mental Disorders, 4th ed. (DSM-IV-TR). Washington, DC: American Psychiatric Association, 1994.

Antshel KM, Hargrave TM, Simonescu M, Kaul P, Hendricks K, Faraone SV: Advances in understanding and treating ADHD. BMC Med 9:72, 2011.

Biederman J, Faraone SV: Attention-deficit hyperactivity disorder. Lancet 366:237-248, 2005.

Busner J, Targum SD: The clinical global impressions scale: Applying a research tool in clinical practice. Psychiatry (Edgmont) 4:28-37, 2007.

Chavez B, Sopko MA, Jr., Ehret MJ, Paulino RE, Goldberg KR, Angstadt K, Bogart GT: An update on central nervous system stimulant formulations in children and adolescents with attention-deficit/ hyperactivity disorder. Ann Pharmacother 43:1084-1095, 2009. 
Childress AC, Brams M, Cutler AJ, Kollins SH, Northcutt J, Padilla A, Turnbow JM: The efficacy and safety of evekeo, racemic amphetamine sulfate, for treatment of attention-deficit/hyperactivity disorder symptoms: a multicenter, dose-optimized, double-blind, randomized, placebo-controlled crossover laboratory classroom study. J Child Adolesc Psychopharmacol 25:402-414, 2015b.

Childress AC, Stark JG, McMahen R, Tengler M, Sikes CR: The Pharmacokinetic Properties of a Novel Extended-Release Oral Disintegrating Tablet Formulation of Methylphenidate in Children and Adolescents with Attention-Deficit/Hyperactivity Disorder. San Antonio, TX: American Academy of Child and Adolescent Psychiatry, 2015a.

Faraone SV, Biederman J, Spencer TJ, Aleardi M: Comparing the efficacy of medications for ADHD using meta-analysis. MedGenMed 8:4, 2006.

Faraone SV, Sergeant J, Gillberg C, Biederman J: The worldwide prevalence of ADHD: Is it an American condition? World Psychiatry 2:104-113, 2003.

Feldman M, Belanger S: Extended-release medications for children and adolescents with attention-deficit hyperactivity disorder. Paediatr Child Health 14:593-602, 2009.

Harpin VA: The effect of ADHD on the life of an individual, their family, and community from preschool to adult life. Arch Dis Child 90 Suppl 1:i2-i7, 2005.

ICH: ICH Harmonised Tripartite Guideline; guideline for good clinical practice E6. International Conference on Harmonisation 1996.

Kieling C, Kieling RR, Rohde LA, Frick PJ, Moffitt T, Nigg JT, Tannock R, Castellanos FX: The age at onset of attention deficit hyperactivity disorder. Am J Psychiatry 167:14-16, 2010.

Nagaraju T, Gowthami R, Rajashekar M, Sandeep S, Mallesham M, Sathish D, Kumar YS: Comprehensive review on oral disintegrating films. Curr Drug Deliv 10:96-108, 2013.

Pliszka S; AACAP Work Group on Quality Issues: Practice parameter for the assessment and treatment of children and adolescents with attention-deficit/hyperactivity disorder. J Am Acad Child Adolesc Psychiatry 46:894-921, 2007.

Polaha J, Dalton WT, 3rd, Lancaster BM: Parental report of medication acceptance among youth: Implications for everyday practice. South Med J 101:1106-1112, 2008.

Polanczyk G, de Lima MS, Horta BL, Biederman J, Rohde LA: The worldwide prevalence of ADHD: A systematic review and metaregression analysis. Am J Psychiatry 164:942-948, 2007.

Posner K, Brown GK, Stanley B, Brent DA, Yershova KV, Oquendo MA, Currier GW, Melvin GA, Greenhill L, Shen S, Mann JJ: The Columbia-Suicide Severity Rating Scale: Initial validity and internal consistency findings from three multisite studies with adolescents and adults. Am J Psychiatry 168:1266-1277, 2011.

Punja S, Zorzela L, Hartling L, Urichuk L, Vohra S: Long-acting versus short-acting methylphenidate for paediatric ADHD: A systematic review and meta-analysis of comparative efficacy. BMJ Open 3:e002312, 2013.

Ramtvedt BE, Aabech HS, Sundet K: Minimizing adverse events while maintaining clinical improvement in a pediatric attentiondeficit/hyperactivity disorder crossover trial with dextroamphetamine and methylphenidate. J Child Adolesc Psychopharmacol 24:130-139, 2014.

Robb AS, Findling RL, Childress AC, Berry SA, Belden HW, Wigal SB: Efficacy, safety, and tolerability of a novel methylphenidate extended-release oral suspension (MEROS) in ADHD. J Atten Disord 2014 [Epub ahead of print]; DOI: 10.1177/1087054714533191.

Saharan VA, Singh A: Fast dissolving/disintegrating dosage forms: Growth from immediate release to sustained release and traditional medicines. Recent Pat Drug Deliv Formul 9:189-191, 2015.

Silva RR, Muniz R, Pestreich L, Childress A, Brams M, Lopez FA, Wang J: Efficacy and duration of effect of extended-release dexmethylphenidate versus placebo in schoolchildren with attention-deficit/hyperactivity disorder. J Child Adolesc Psychopharmacol 16:239-251, 2006.

Subcommittee on Attention-Deficit/Hyperactivity Disorder; Steering Committee on Quality Improvement and Management; Wolraich M, Brown L, Brown RT, DuPaul G, Earls M, Feldman HM, Ganiats TG, Kaplanek B, Meyer B, Perrin J, Pierce K, Reiff M, Stein MT, Visser S: ADHD: Clinical practice guideline for the diagnosis, evaluation, and treatment of attention-deficit/hyperactivity disorder in children and adolescents. Pediatrics 128:1007-1022, 2011.

Sugrue D, Bogner R, Ehret MJ: Methylphenidate and dexmethylphenidate formulations for children with attention-deficit/hyperactivity disorder. Am J Health Syst Pharm 71:1163-1170, 2014.

Swanson J, Gupta S, Lam A, Shoulson I, Lerner M, Modi N, Lindemulder E, Wigal S: Development of a new once-a-day formulation of methylphenidate for the treatment of attention-deficit/ hyperactivity disorder: Proof-of-concept and proof-of-product studies. Arch Gen Psychiatry 60:204-211, 2003.

Swanson J, Kinsbourne M, Roberts W, Zucker K: Time-response analysis of the effect of stimulant medication on the learning ability of children referred for hyperactivity. Pediatrics 61:21-29, 1978.

Swanson JM, Wigal S, Greenhill LL, Browne R, Waslik B, Lerner M, Williams L, Flynn D, Agler D, Crowley K, Fineberg E, Baren M, Cantwell DP: Analog classroom assessment of Adderall in children with ADHD. J Am Acad Child Adolesc Psychiatry 37:519-526, 1998.

Thomas R, Sanders S, Doust J, Beller E, Glasziou P: Prevalence of attention-deficit/hyperactivity disorder: A systematic review and meta-analysis. Pediatrics 135:e994-e1001, 2015.

Visser SN, Bitsko RH, Danielson ML, Ghandour RM, Blumberg SJ, Schieve LA, Holbrook JR, Wolraich ML, Cuffe SP: Treatment of attention deficit/hyperactivity disorder among children with special health care needs. J Pediatr 166:1423-1430.e1-2, 2015.

Visser SN, Danielson ML, Bitsko RH, Holbrook JR, Kogan MD, Ghandour RM, Perou R, Blumberg SJ: Trends in the parent-report of health care provider-diagnosed and medicated attention-deficit/ hyperactivity disorder: United States, 2003-2011. J Am Acad Child Adolesc Psychiatry 53:34-46.e2, 2014.

Wigal SB, Childress AC, Belden HW, Berry SA: NWP06, an extended-release oral suspension of methylphenidate, improved attention-deficit/hyperactivity disorder symptoms compared with placebo in a laboratory classroom study. J Child Adolesc Psychopharmacol 23:3-10, 2013.

Wigal SB, Gupta S, Guinta D, Swanson JM: Reliability and validity of the SKAMP rating scale in a laboratory school setting. Psychopharmacol Bull 34:47-53, 1998.

Wigal SB, Kollins SH, Childress AC, Squires L, Study G: A 13-hour laboratory school study of lisdexamfetamine dimesylate in schoolaged children with attention-deficit/hyperactivity disorder. Child Adolesc Psychiatry Ment Health 3:17, 2009.

Wigal SB, Wigal TL: The laboratory school protocol: Its origin, use, and new applications. J Atten Disord 10:92-111, 2006.

World Medical Association: World Medical Association Declaration of Helsinki: Ethical principles for medical research involving human subjects. JAMA 310:2191-2194, 2013.

Address correspondence to: Carolyn Sikes, PhD Vice President of Clinical Affairs Neos Therapeutics, Inc.

2940 N. Highway 360 Grand Prairie, TX 75050

E-mail: csikes@neostx.com 